\title{
Self-triggered rendezvous of gossiping second-order agents
}

\author{
Claudio De Persis
}

\author{
Paolo Frasca
}

\author{
Julien M. Hendrickx
}

\begin{abstract}
A recent paper by some of the authors introduced several self-triggered coordination algorithms for first-order continuous-time systems. The extension of these algorithms to second-order agents is relevant in many practical applications but presents some challenges that are tackled in this contribution and that require to depart from the analysis that was carried out before. We design a self-triggered gossiping coordination algorithm that induces a time-varying communication graph, which is enough connected to guarantee useful convergence properties, and allows us to achieve the desired coordination task in a formation of double-integrator agents that (i) establish pair-wise communication at suitably designed times and (ii) exchange relative measurements while reducing the sensing and communication effort.
\end{abstract}

\section{INTRODUCTION}

In a number of recent works [12], [18], [7], [6], [19], [5], authors have been focusing on cooperative control problems in which the agents do not exchange information continuously but rather at times that are decided based on current local measurements. These coordination strategies can be of two types: event-triggered or self-triggered coordination control. The focus of this paper is on the second class of coordination control algorithms and is inspired by the socalled self-triggered gossiping algorithms of [6].

The term "self-triggered gossiping" refers to the way in which agents establish communication: each pair of agents connected by an edge of an undirected graph decide in realtime the next sampling time based on the local measurements. The design of these sampling times by a pair of connected nodes is performed in a completely independent way from the other pairs and results in an asynchronous information exchange pattern. Moreover, this approach can lead to a significant reduction of communication and sensor activity: at each sampling time the sensors of the connected agents switch to active mode, exchange measurements, compute the next sampling time and then go to sleep mode until the next sampling time is reached.

Claudio De Persis is with Faculty of Mathematics and Natural Sciences, University of Groningen, the Netherlands, c.de.persis@rug.nl. His work is partially supported by the Dutch Organization for Scientific Research (NWO) under the auspices of the project QUICK (QUantized Information Control for formation Keeping).

Paolo Frasca was with Dipartimento di Scienze Matematiche, Politecnico di Torino, Torino, Italy and is now with Department of Applied Mathematics, University of Twente, 7500 AE Enschede, The Netherlands, p.frasca@utwente.nl.

Julien M. Hendrickx is with ICTEAM Institute, Université catholique de Louvain, B-1348 Louvain-la-Neuve, Belgium, julien.hendrickx@uclouvain.be. His work is supported by the Belgian Network DYSCO (Dynamical Systems, Control, and Optimization), funded by the Interuniversity Attraction Poles Program, initiated by the Belgian Science Policy Office. The research is also supported by the Concerted Research Action (ARC) Large Graphs and Networks of the French Community of Belgium.
The focus in [6] was on first-order multi-agent systems. Nevertheless, as argued in [2], [16], in the majority of coordination problems neglecting the dynamics of the systems is not possible. In this paper we take a first step towards the design of self-triggered gossiping algorithms for second-order linear systems: we design and analyze a self-triggered coordination control algorithm that achieves practical rendezvous of double-integrator agents with guaranteed dwell-time. By practical rendezvous it is meant that the agents converge to a neighborhood of the rendezvous point, where the size of the neighborhood can be tuned at will by a parameter in the controller. This parameter also regulates the value of the induced dwell-time. Although the rendezvous problem for double integrators that we treat here represents a very special class of coordination problems for multi-agent systems, it is usually a useful starting point for understanding more general problems that include, e.g., formation achievement, unknown reference velocity recovery and disturbance rejection [2], [16], possibly for agents with nonlinear dynamics.

Besides the extension to a more general class of multiagent systems, one of the main contributions of this work is the link that we establish between event-based coordination control and a recently proved sufficient condition for the convergence of consensus algorithms, involving very general cut-balance conditions [8]. Indeed, one of the difficulties that we face when going from first-order to second-order agents is that the Lyapunov-based analysis that was pursued in [6] can not be directly extended. Instead of insisting on the search for an appropriate Lyapunov function (a topic that could nevertheless be of interest for future research), we pursue here a different approach relying on the results of [8].

\section{Comparison with existing literature}

This paper enlarges the class of systems for which the selftriggered distributed control proposed in [6] applies. In doing so, it explores a new investigation line based on the cutbalance condition of [8]. The current manuscript still exhibits a few of the features of the approach in [6], which makes the contribution different from those in [18] and [7]. In brief, when compared with [18], our approach requires polling neighbors rather than broadcasting information to them, a feature that was inspired by [12], where it was employed in problems of deployment for agents with dynamics different from those considered in our paper. With respect to [7], relative measurements of single-hop neighbors are used and a guaranteed dwell-time is ensured. Other works that focus on event-triggering cooperative control are [5], that deals with event-triggered practical synchronization of linear systems, and [19], where event-triggered output synchronization of 
passive systems is studied. The main difference of our work with [5] and [19] lies in the approach chosen to design the triggering functions.

Our approach is inspired by non-smooth coordination algorithms [4], [9] in which the task is achieved using binary information. This suggests that coordination does not require very precise information. Our triggering functions are designed in such a way that information is collected only when measurements are expected to change their signs. As a secondary result of this design, the term in the control that is due to inter-agent interaction takes value in a finite set, a feature that simplifies the analysis of the overall system.

Linear consensus systems have been the object of numerous studies, both in discrete and continuous time (see for example the surveys [15], [17]). Typical results establish convergence to exact consensus provided that the graph describing the interactions remains always connected, or satisfies some so-called repeated connectivity condition. The specific aspect that interests us here is that, under some weak form of symmetry of the interactions, convergence (though not necessarily to consensus) is guaranteed independently of the connectivity of the interaction graph. Moreover, simple conditions for two agents to converge to the same value are then available. These results were established for continuoustime system in [8]. Related observations for discrete-time systems were made in [3], [11], [10], (see also references in [8]).

\section{Paper organization}

The paper is organized as follows. In Section II, we discuss about the motivation for our work. In Section III we introduce the algorithm as a hybrid system and comment on a few of its features. In Section IV the main convergence result is stated and proved. Numerical simulations are discussed in Section V. The last section is devoted to the conclusions and to a few directions for future research.

\section{Motivation: Rendezvous of SECOND-ORDER AGENTS}

We consider a set of double integrators

$$
\begin{aligned}
\dot{p}_{i} & =v_{i} \\
\dot{v}_{i} & =\tau_{i}, \quad i \in I:=\{1, \ldots, n\}
\end{aligned}
$$

with $p_{i}, v_{i}, \tau_{i} \in \mathbb{R}$, distributed over a connected graph $\mathcal{G}=$ $(I, E)$, where $E=\{1,2, \ldots, m\}$ is the set of the edges. We let $A=\left[a_{i j}\right]$ be a weighted adjacency matrix associated to the graph, with $a_{i i}=0$ for all $i \in I$ and $a_{i j}=0$ if $j \notin \mathcal{N}_{i}$, where $\mathcal{N}_{i}=\{j \in I:(i, j) \in E\}$ is the set of the neighbors of $i$. We assume the matrix $A$ to be symmetric, i.e., we assume $a_{i j}=a_{j i}$ for all $i, j$ in $I$.

For the distributed system above, several coordination problems can be formulated. In this paper we focus on a rendezvous problem in which the agents converge to a common point with velocity which asymptotically vanishes. More complex coordination problems can be formulated, as discussed for instance in [2]. If for the sake of simplicity we restrict ourselves to the case in which the non-zero entries of the adjacency matrix are equal to 1 (this condition will be removed starting from Section III), the rendezvous problem for the system (1) is known (see e.g. [1]) to be solvable by the distributed feedback law

$$
\tau_{i}=-2 g v_{i}+\sum_{j=1}^{n} a_{i j}\left(p_{j}-p_{i}\right)
$$

for any positive gain $g$. Furthermore, under this law the agents rendezvous to a point which can be computed as a function of the initial conditions. Indeed, the closed-loop system is described by the compact form

$$
\begin{aligned}
\dot{p} & =v \\
\dot{v} & =-2 g v-L p .
\end{aligned}
$$

If we denote the average of a vector $y \in \mathbb{R}^{n}$ as ave $(y)=$ $\frac{1}{n} \sum_{i=1}^{n} y_{i}$, we observe that the average of positions and velocities satisfies

$$
\begin{aligned}
& \frac{d}{d t} \operatorname{ave}(p(t))=\operatorname{ave}(v(t)) \\
& \frac{d}{d t} \operatorname{ave}(v(t))=-2 g \operatorname{ave}(v(t)) .
\end{aligned}
$$

Then, integration yields that

$$
\operatorname{ave}(p(t))=\operatorname{ave}(p(0))+\frac{1-\mathrm{e}^{-2 g t}}{2 g} \operatorname{ave}(v(0))
$$

and taking the limit as $t \rightarrow \infty$, it gives that $p^{*}=\operatorname{ave}(p(0))+$ $\frac{1}{2 g} \operatorname{ave}(v(0))$.

Continuous-time distributed control algorithms like (2) are well-known in the literature. Less attention has been paid to algorithms which do not require a continuous exchange of information among the agents. In this paper we investigate a variation of (2) in which agents communicate in a pairwise fashion at instants that are determined in real-time. The motivation to the design of the algorithm presented below originates in the following result that is established in [9].

Proposition 1 In the case $a_{i j} \in\{0,1\}$, for any $g>0$, all the Krasowskii solutions to

$$
\begin{aligned}
& \dot{p}_{i}=v_{i} \\
& \dot{v}_{i}=-2 g v_{i}-\sum_{j=1}^{n} a_{i j} \operatorname{sign}\left(p_{j}-p_{i}\right), \quad i \in I
\end{aligned}
$$

where $\operatorname{sign}(\cdot): \mathbb{R} \rightarrow\{-1,0,+1\}$ is the function

$$
\operatorname{sign}(p)=\left\{\begin{array}{rl}
+1 & p>0 \\
0 & p=0 \\
-1 & p<0
\end{array}\right.
$$

converge to the point $\left(p^{*}, 0\right)$.

We omit the definition of Krasowskii solutions for which we refer the reader to e.g. [9]. We stress that the result above points out that to achieve the coordination task the controllers must only keep track of the zero-crossing events occurring to the relative position $p_{i}-p_{j}$. The algorithm that we introduce in the next section exploits this observation and devises a strategy that achieves rendezvous by activating the position sensors only at times when $p_{i}-p_{j}$ is expected to reach zero. 


\section{SELF-TRIGGERED GOSSIPING}

Following [6], the proposed consensus algorithm is described by a network of hybrid systems. This choice allows us to present the algorithm in a concise yet expressive way. We focus on a hybrid algorithm to achieve consensus for double integrators inspired by the so-called self-triggered gossiping algorithm of [6] that was proposed for multi-agent systems modeled as single integrators.

We begin by introducing the additional states necessary to the agents for implementing our algorithm. For each agent $i$ and neighbor $j$ of $i$, we let $u_{i}^{j} \in\{-1,0,1\}$ be a ternary state that represents the sign (up to a threshold $\varepsilon$ ) that $p_{j}-p_{i}$ had the last time that it was measured, and $\theta_{i}^{j} \in \mathbb{R}_{\geq 0}$ be the remaining time until the next measurement of $p_{j}-p_{i}$. Our algorithm will ensure that $\theta_{j}^{i}=\theta_{i}^{j}$, so that $i$ measures $p_{j}-p_{i}$ at the same time as $j$ measures $p_{i}-p_{j}$, and $u_{i}^{j}=-u_{j}^{i}$. The state vector for each agent is thus

$$
\xi_{i}=\left(p_{i}, v_{i}, u_{i}^{1}, \ldots, u_{i}^{n_{i}}, \theta_{i}^{1}, \ldots, \theta_{i}^{n_{i}}\right),
$$

where $n_{i}$ denotes the degree of node $i$, that is the number of its neighbors.

The algorithm is described by the following dynamics. For each $i \in I$, for all times at which $\theta_{i}^{j}>0$ for every neighbor $j$ of $i$,

$$
\left\{\begin{aligned}
\dot{p}_{i} & =v_{i} \\
\dot{v}_{i} & =-2 g v_{i}+\sum_{j=1}^{n} a_{i j} u_{i}^{j} \\
\dot{u}_{i}^{j} & =0, \quad j \in \mathcal{N}_{i} \\
\dot{\theta}_{i}^{j} & =-1, \quad j \in \mathcal{N}_{i}
\end{aligned}\right.
$$

and for times at which $\theta_{i}^{j}=0$ for at least one neighbor $j$ of $i$, i.e., times at which a measurement is made,

$$
\left\{\begin{aligned}
p_{i}^{+} & =p_{i} \\
v_{i}^{+} & =v_{i} \\
u_{i}^{j+} & = \begin{cases}\operatorname{sign}_{\varepsilon}\left(p_{j}(t)-p_{i}(t)\right) & \text { if } \theta_{i}^{j}=0 \\
u_{i}^{j}(t) & \text { otherwise }\end{cases} \\
\theta_{i}^{j+} & = \begin{cases}f_{i}^{j}(p(t), v(t)) & \text { if } \theta_{i}^{j}=0 \\
\theta_{i}^{j}(t) & \text { otherwise }\end{cases}
\end{aligned}\right.
$$

where $f_{i}^{j}$ is an appropriate mapping defined below, and sign ${ }_{\varepsilon}$ denotes a sign function with a threshold $\varepsilon$ (which is a design parameter):

$$
\operatorname{sign}_{\varepsilon}(x)=\left\{\begin{array}{cl}
+1 & x>\varepsilon \\
0 & |x| \leq \varepsilon \\
-1 & x<-\varepsilon
\end{array}\right.
$$

The algorithm is initialized in such a way that $\theta_{i}^{j}(0)=\theta_{j}^{i}(0)$ and $u_{i}^{j}(0)=-u_{j}^{i}(0)$ for all $(i, j) \in E$. These assumptions guarantee that $\theta_{i}^{j}$ and $\theta_{j}^{i}$ remain equal for all times, and therefore that $u_{i}^{j}=-u_{j}^{i}$. However, in the following analysis we assume $\theta_{i}^{j}(0)$, so that all controls are updated at time zero: while our main statements also apply to the general case, derivations become more involved.

The following lemma introduces a mapping $f_{i}^{j}$ guaranteeing that (i) the periods of time between two measurements of the same relative position remain larger than a certain positive bound, and (ii) if two agents are separated by more than $\varepsilon$ when they measure their relative positions, they do not cross (i.e., their relative position keeps the same sign) before the next measurement. Its proof can be found in the Appendix.

\section{Lemma 1 Define}

$$
f_{i}^{j}(p, v)=\left\{\begin{array}{lll}
\frac{\left|p_{i}-p_{j}\right|}{2\left(\left|v_{i}-v_{j}\right|+\frac{d_{i}+d_{j}}{2 g}\right)} & \text { if } & \left|p_{i}-p_{j}\right| \geq \varepsilon \\
\frac{\varepsilon}{2\left(\left|v_{i}-v_{j}\right|+\frac{d_{i}+d_{j}}{2 g}\right)} & \text { if } & \left|p_{i}-p_{j}\right|<\varepsilon,
\end{array}\right.
$$

where we denote $d_{k}=\sum_{\ell=1}^{n} a_{k \ell}$. For a given trajectory of (5), (6) with the mappings $f_{i}^{j}$ as above, define for each pair of connected nodes $(i, j)$ the sequence of consecutive measurement times $t_{m}^{i j}$ by $t_{0}^{i j}=0$ and $t_{m+1}^{i j}=t_{m}^{i j}+f_{i}\left(p\left(t_{m}^{i j}\right), v\left(t_{m}^{i j}\right)\right)$.

(i) For all $m$ it holds true that

$$
t_{m+1}^{i j}-t_{m}^{i j} \geq \frac{\varepsilon}{2\left(\left|v_{i}(0)-v_{j}(0)\right|+\frac{d_{i}+d_{j}}{2 g}\right)} .
$$

(ii) If $\left|p_{j}\left(t_{m}^{i j}\right)-p_{i}\left(t_{m}^{i j}\right)\right| \geq \varepsilon$, then for all $t \in\left[t_{m}^{i j}, t_{m+1}^{i j}\right]$, there holds

$$
\left|p_{j}(t)-p_{i}(t)\right| \geq \frac{\left|p_{j}\left(t_{m}^{i j}\right)-p_{i}\left(t_{m}^{i j}\right)\right|}{2},
$$

and $\operatorname{sign}\left(p_{j}(t)-p_{i}(t)\right)=\operatorname{sign}\left(p_{j}\left(t_{m}^{i j}\right)-p_{i}\left(t_{m}^{i j}\right)\right)$.

A few comments are in order after this result.

Remark 1 (Dwell time) By definition of $\theta_{i}^{j+}$, after agent $i$ has collected the measurements about its neighbor $j$ at time $t$, at least $f_{i}^{j}(p(t), v(t))$ units of time must elapse before the agents polls again the neighbor. In view of (8), this event does not occur earlier than

$$
\frac{\varepsilon}{2\left(\left|v_{i}(0)-v_{j}(0)\right|+\frac{d_{i}+d_{j}}{2 g}\right)}
$$

units of time. In other words, given a compact set of initial velocities of the formation, for any pair of agents connected by a link in the graph $\mathcal{G}$, there exists a minimal inter-sampling time between two consecutive information exchange between these two agents. This is related to the notion of uniform semi-global dwell-time available in the event-based control and discussed e.g. in [13]. Moreover, since (5) evolves as a linear system during continuous evolution, the solution to (5), (6) exists over each interswitching interval. As no accumulation of switching times in finite time exists, then the solution to (5), (6) exists for all $t$ and is unique. In particular, the sequence $t_{m}^{i j}$ defined in Lemma 1 is divergent, and contains all the sampling times of the position of $j$ relative to $i$ and vice-versa.

Remark 2 (Relative measurements) Consistently with the control law (2), the piecewise constant term defined by $u_{i}^{j}$ only requires to measure the inter-agent relative position $p_{j}-$ $p_{i}$. Nevertheless, in order to compute the next sampling time, the agent $i$ needs information on the magnitude of both the relative position $p_{j}-p_{i}$ and the relative velocity $v_{i}-v_{j}$. 
Remark 3 (Use of local information) In view of (5), the control that each agent $i$ applies is $\tau_{i}=-2 g v_{i}+\sum_{j=1}^{n} a_{i j} u_{i}^{j}$. The first term is a damping term that depends on a quantity that is available to agent $i$ (its velocity). As such, this information has not to be retrieved from the neighboring agents, and it is assumed that it is available continuously at each time. The second term is a consensus-like term that depends on information related to neighboring agents. To reduce the interaction between the agents, this information is acquired only at the sampling times.

Remark 4 (Synchrony of clock variables) As we anticipated, the clock variables $\theta_{i}^{j}$ and $\theta_{j}^{i}$ are equal for all time. This is a consequence of the chosen initialization and of the definition of $f_{i}^{j}(p, v)$ in which $\left|p_{i}-p_{j}\right|$ and $\left|v_{i}-v_{j}\right|$ appear.

Remark 5 (Dynamics of the average) Formula (3) also holds for system (5), (6), because $a_{i j}=a_{j i}$ for every $i, j \in I$.

\section{Analysis AND MaIn Result}

The hybrid system (5), (6) is useful to properly define the consensus algorithm that we are investigating and to explain how the agents collect information from their neighbors. However, to the purpose of analyzing the convergence properties of the system, we find convenient to "embed" it into a first-order consensus algorithm as in [14]. This is possible (for large enough $g$ ) because the $p, v$ state variables remain unchanged during discrete evolution. We denote by $t_{m}^{i j}$ the largest sampling time smaller than $t$, i.e., $t_{m}^{i j}=\max \left\{t_{\ell}^{i j}: t_{\ell}^{i j}<\right.$ $t$, for all $m \in \mathbb{N}$ (a slightly abusive notation since $t_{m}^{i j}$ is really a function of $t$ ). Then, for all $t \in\left[t_{m}^{i j}, t_{m+1}^{i j}\right]$ the evolution of $p, v$ is equivalently described by

$$
\begin{aligned}
& \dot{p}_{i}(t)=v_{i}(t) \\
& \dot{v}_{i}(t)=-2 g v_{i}(t)+\sum_{j=1}^{n} a_{i j} \operatorname{sign}_{\varepsilon}\left(p_{j}\left(t_{m}^{i j}\right)-p_{i}\left(t_{m}^{i j}\right)\right) .
\end{aligned}
$$

The following Lemma, whose proof can be found in the Appendix, provides a change of variables which is instrumental to our main result.

\section{Lemma 2 Define}

$$
\beta_{i j}(p(t))=\left\{\begin{array}{cccc}
\frac{a_{i j}}{\left|p_{j}(t)-p_{i}(t)\right|} & \text { if } & \left|p_{j}\left(t_{m}^{i j}\right)-p_{i}\left(t_{m}^{i j}\right)\right| \geq \varepsilon \\
0 & \text { if } & \left|p_{j}\left(t_{m}^{i j}\right)-p_{i}\left(t_{m}^{i j}\right)\right|<\varepsilon .
\end{array}\right.
$$

Then, for $i, j \in I$, the coefficients $\beta_{i j}(x(t))$ are non-negative and such that for any trajectory there exists a $\beta_{0}>0$ for which $\beta_{i j}(x(t))$ is either 0 or larger than $\beta_{0}$.

Assume that $g \geq \sqrt{\frac{2 d_{i}}{\varepsilon}}$ for $i \in I$, where $d_{i}=\sum_{j=1}^{n} a_{i j}$. Then, the change of coordinates [14]

$$
\begin{aligned}
y_{i} & =\frac{1}{g} v_{i}+p_{i}, \quad i \in I \\
x_{2 i-1} & =p_{i}, \quad x_{2 i}=y_{i}, \quad i \in I
\end{aligned}
$$

transforms system (10) into system

$$
\dot{x}_{h}=\sum_{k=1}^{2 n} \alpha_{h, k}\left(x_{k}-x_{h}\right)
$$

where the coefficients $\alpha_{h, k}$ are

$$
\begin{gathered}
\alpha_{2 i-1,2 i}(t)=g, \quad \alpha_{2 i, 2 i-1}(t)=g-\frac{1}{g} \sum_{k=1}^{n} \beta_{i k}(p(t)), \\
\alpha_{2 i, 2 j-1}(t)=\frac{1}{g} \beta_{i j}(p(t)), \quad j \neq i,
\end{gathered}
$$

and all others are zero. These coefficients $\alpha_{h, k}$ are nonnegative and can be seen as the entries of the adjacency matrix of a weight-balanced graph $\tilde{G}$ (i.e., $\sum_{k} \alpha_{h, k}=\sum_{k} \alpha_{k, h}$ ) with node set $\tilde{I}=\{1, \ldots, 2 n\}$.

This lemma allows us to transform the network of secondorder dynamics in (10) into a network of first-order dynamics, and then to apply the convergence criteria in [8] on the resulting graph $\tilde{G}$. We are then ready to prove the main result of this paper.

Theorem 1 Consider the self-triggered gossiping algorithm (5), (6) where $a_{i j}=a_{j i}$ are the entries of the adjacency matrix associated to a connected graph $\mathcal{G}=(I, E), \varepsilon>0$ is an arbitrary constant, and $g$ satisfies $g \geq \sqrt{\frac{2 d_{i}}{\varepsilon}}$ for $i \in\{1, \ldots, n\}$. Assume that $\theta(0)=\mathbf{0}$. Then, the solution to (5), (6) converges asymptotically to a point in

$$
\left\{(p, v):\left|p_{j}-p_{i}\right| \leq \varepsilon \forall(i, j) \in E, v=\mathbf{0}\right\} .
$$

Moreover, there exists a finite time $T$ such that $\left|p_{j}(t)-p_{i}(t)\right| \leq$ $\varepsilon$ and $u_{i}^{j}(t)=0$ for all $t>T$ and $(i, j) \in E$.

Proof: Having shown in Lemma 2 that the weighted graph $\tilde{\mathcal{G}}$ corresponding to the first-order consensus algorithm (13) is weight-balanced, by Proposition 1(c) in [8] we can conclude that it satisfies the more general cut-balance condition therein, and therefore that Theorem 1 from the same paper applies. It follows from this theorem that (i) all $x_{k}$ converge, i.e. $x_{k}^{*}=\lim _{t \rightarrow \infty} x_{k}(t)$ exists for every $k$, and (ii) $\int_{t=0}^{\infty} \alpha_{k h}(s) d s=\infty$ implies $x_{k}^{*}=x_{h}^{*}$. Observe that for any $i \in\{1, \ldots, n\}$, the coefficient $\alpha_{2 i-1,2 i}(t)=g$ linking $p_{i}$ to $y_{i}=p_{i}+v_{i} / g$ satisfies the condition $\int_{0}^{+\infty} \alpha_{2 i-1,2 i}(t) d t=\infty$. Therefore, there holds $p_{i}^{*}=x_{2 i-1}^{*}=x_{2 i}^{*}=y_{i}^{*}=p_{i}^{*}+v_{i}^{*} / g$ and thus by (11) $v_{i}^{*}=\lim _{t \rightarrow \infty} v_{i}(t)=0$.

We now show the existence of a time after which all active controls are 0 . Consider a pair of agents $i, j$ connected in the graph $\mathcal{G}$ and suppose, to obtain a contradiction, that there is a diverging sequence of times at which $u_{i}^{j}$ is nonzero. Observe that by (5) and (6), $u_{i}^{j}$ can only change from zero to nonzero and vice-versa at the measurement times $t_{i j}^{m}$, which were shown in Lemma 1 to differ from each other by at least some positive lower bound. $u_{i}^{j}$ must thus be positive on infinitely many intervals of length larger than this lower bound. Moreover, whenever $u_{i}^{j}$ is positive, it follows from Lemma 2 that $\alpha_{2 i, 2 j-1}(t) \geq \frac{\beta_{0}}{g}$. So there must hold $\int_{0}^{\infty} \alpha_{2 i, 2 j-1}(t) d t=\infty$, and therefore (by (ii) above) $y_{i}^{*}=x_{2 i}^{*}=x_{2 j-1}^{*}=p_{j}^{*}$. Remembering that $y_{i}^{*}=p_{i}^{*}$, this implies that $\left|p_{i}(t)-p_{j}(t)\right|$ converges to 0 , and remains thus smaller than $\varepsilon$ after a certain time $\vec{t}^{i j}$. But by the definition of our algorithm in (5) and (6), $u_{i}^{j}$ becomes then 0 after the first 
measurement time $t_{m}^{i j}$ that follows $\vec{t}^{i j}$, in contradiction with our assumption that it is nonzero on a diverging sequence of times. Thus, there is a time $T^{\prime}$ after which $u_{i}^{j}(t)=0$ for all $t>T^{\prime}$.

Having eventually zero controls implies that $\left|p_{i}^{*}-p_{j}^{*}\right| \leq \varepsilon$ for all $(i, j) \in E$, for otherwise the continuity of $p_{i}-p_{j}$ and the definition of $u_{i}^{j}$ would imply that $u_{i}^{j}$ always remains positive after a certain time. Moreover, after the time $T^{\prime}$, since $u=0$, each agent behaves autonomously according to $\dot{p}_{i}=v_{i}, \dot{v}_{i}=$ $-2 g v_{i}$. Solving for the velocity yields $v_{i}(t)=v_{i}(T) e^{-2 g(t-T)}$, whose integration leads to $p_{i}(t)=K_{i}-\frac{v_{i}(T)}{2 g} e^{-2 g(t-T)}$ for some constant $K_{i}$. Taking the limit, we see that $\lim _{t \rightarrow \infty} p_{i}(t)=K_{i}$, and therefore that $K_{i}=p_{i}^{*}$, so that $p_{i}(t)=p_{i}^{*}-\frac{v_{i}(T)}{2 g} e^{-2 g(t-T)}$. As a consequence, each relative position $p_{j}(t)-p_{i}(t)=p_{j}^{*}-$ $p_{i}^{*}-e^{-2 g(t-T)} \frac{v_{j}(T)-v_{i}(T)}{2 g}$ evolves monotonously. Since we have already proved for any $(i, j) \in E$ that there holds $\left|p_{j}^{*}-p_{i}^{*}\right| \leq \varepsilon$ and that $\left|p_{j}\left(t_{m}^{i j}\right)-p_{i}\left(t_{m}^{i j}\right)\right| \leq \varepsilon$ at every measurement time $t_{m}^{i j}>T^{\prime}$, this monotonicity implies that $\left|p_{j}(t)-p_{i}(t)\right| \leq \varepsilon$ holds for all $t>T$ for some $T>T^{\prime}$.

As opposed to Theorem 1, we recall that in (4), the gain $g$ can be any positive number. Actually, the numerical study in the next section shows that convergence occurs even if $g$ is below the threshold given in the statement above. In fact, the condition on $g$ is only sufficient and is a technically assumption needed to guarantee that the induced graph $\tilde{\mathcal{G}}$ is weight-balanced. Proving convergence while lifting this assumption remains an interesting open question.

\section{Simulations}

We have simulated the proposed system (5), (6) using cycle and line graphs of different sizes, starting from random initial conditions sampled from uniform distributions. Simulations confirm the insights from our theoretical analysis. Specifically, we report about the case of a line graph with five nodes, choosing three different values of the gain $g$. The interest in line graphs depends on their natural occurrence in one-dimensional problems, e.g., involving platoons of vehicles moving on a straight line. As expected, simulations show that positions converge asymptotically to a configuration which is close to rendezvous, while velocities converge to zero. At steady state the positions of consecutive agents differ by no more than $\varepsilon$, in full accordance with Theorem 1 . Consistently, the components of the active controls that are due to the inter-agent interaction become zero after a finite time, when rendezvous is approximately reached. After that time velocities exponentially die out.

As we already mentioned, the condition on $g$ does not seem necessary for convergence. Indeed, the simulations depicted in Figures 2 and 3 refer to values of $g$ below the threshold given in Theorem 1, and nevertheless show convergence. Actually, trajectories are smoother and may converge faster for smaller gains. However, if we compare Figures 1 and 2 against Figure 3, we observe that for small enough gains the trajectories may intersect. This aspect asks for further investigation, as in some applications these intersections may represent collisions between agents.

\section{Conclusions}

We have introduced a self-triggered gossiping algorithm to achieve rendezvous in formations of second-order agents. In the analysis we exploit ideas from self-triggered coordination algorithms in combination with tools for consensus under very general conditions. This paper represents an initial step towards a full understanding of the problem, as many questions are left open. In fact, we design and analyze a coordination algorithm for a class of linear multi-agent systems that are connected over an undirected connected graph. The generality of the methods of [8], namely the lack of any assumption on how the coefficients vary (with the exception of the cut-balance condition), makes us think that similar results can be achieved for more general classes of connecting graphs, possibly time-varying.

Robustness is a critical feature for self-triggered control algorithms. In [6] robustness to clock skews, delays and quantization was investigated. We envision a similar study to be developed for the algorithms introduced in this paper. In fact, we expect such a robustness property to hold provided that new triggering functions are designed to guarantee the sign preservation property of Lemma 1 in the presence of uncertainties. We also remark that by an appropriate redesign of the triggering rules, the self-triggered algorithms of [6] achieve also asymptotic coordination. Whether this redesign is possible with the triggering rules introduced in this paper is also a topic for future study.

We have focused on a basic (yet important) coordination task, namely the rendezvous problem. Many other coordination tasks can be investigated, possibly taking into account important features such as collision avoidance. Whether these problems can be solved by the self-triggered coordination controls introduced in this paper is left to future investigation.

\section{REFERENCES}

[1] M. Arcak. Passivity as a design tool for group coordination. IEEE Transactions on Automatic Control, vol. 52, no. 8, pp. 1380-1390, 2007.

[2] H. Bai, M. Arcak, and J. Wen. Cooperative Control Design: A Systematic, Passivity-Based Approach. Communications and Control Engineering. Springer, New York, 2011.

[3] V.D. Blondel, J.M. Hendrickx, A. Olshevsky, and J.N. Tsitsiklis. "Convergence in multiagent coordination, consensus, and flocking". In Proceedings of the 44th IEEE Conference on Decision and Control (CDC2005), Seville, Spain, Dec. 2005, pp. 2996-3000

[4] J. Cortés, "Finite-time convergent gradient flows with applications to network consensus," Automatica, vol. 42, no. 11, pp. 1993-2000, 2006.

[5] O. Demir and J. Lunze, "Event-based synchronisation of multi-agent systems," in 4th IFAC Conference on Analysis and Design of Hybrid Systems (ADHS 12), Santa Barbara, CA, USA, Sep. 2012, pp. 43-48.

[6] C. De Persis and P. Frasca, "Self-triggered coordination with ternary controllers," in IFAC Workshop on Estimation and Control of Networked Systems, Santa Barbara, CA, USA, Sep. 2012, pp. 43-48.

[7] D.V. Dimarogonas, E. Frazzoli, and K.H. Johansson, "Distributed event-triggered control for multi-agent systems," IEEE Transactions on Automatic Control, vol. 57, no. 5, pp. 1291-1297, 2012.

[8] J.M. Hendrickx and J. Tsitsiklis, "Convergence of type-symmetric and cut-balanced consensus seeking systems". In IEEE Transactions on Automatic Control, vol. 58, no. 1, pp. 214-218, 2013.

[9] M. Jafarian and C. De Persis. "Exact formation control with very coarse information". Proceedings of the 2013 American Control Conference, Washington, DC, June 2013.

[10] J. Lorenz. "A stabilization theorem for continuous opinion dynamics". Physica A, vol. 355, no. 1, pp. 217-223, 2005. 

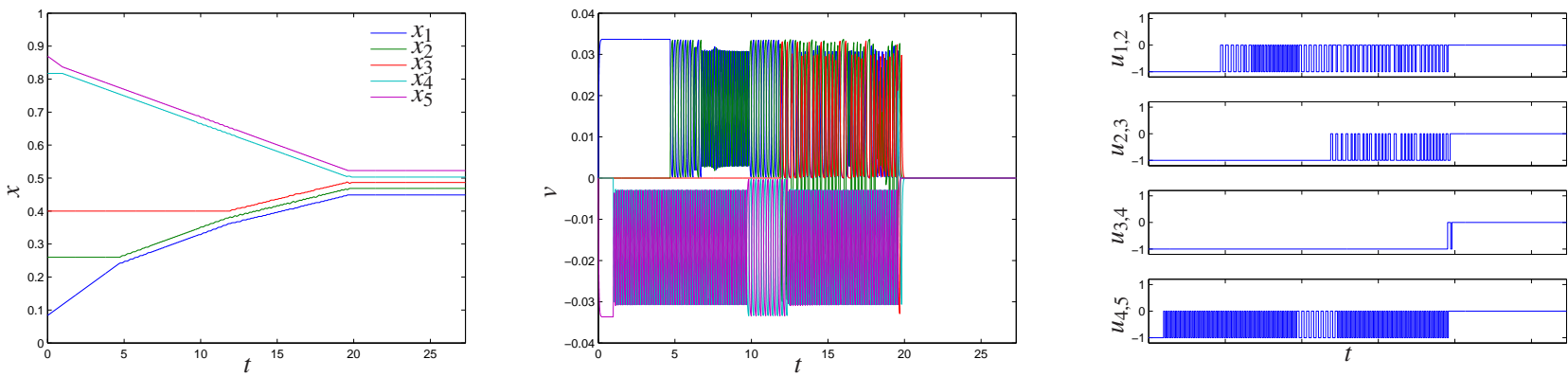

Fig. 1. Simulation of system (5), (6) on a line graph with $n=5$ nodes, $\varepsilon=0.02$, and $g=14.84$. The condition on $g$ in Theorem 1 is satisfied. Initial positions in $[0,1]$, null initial velocities.
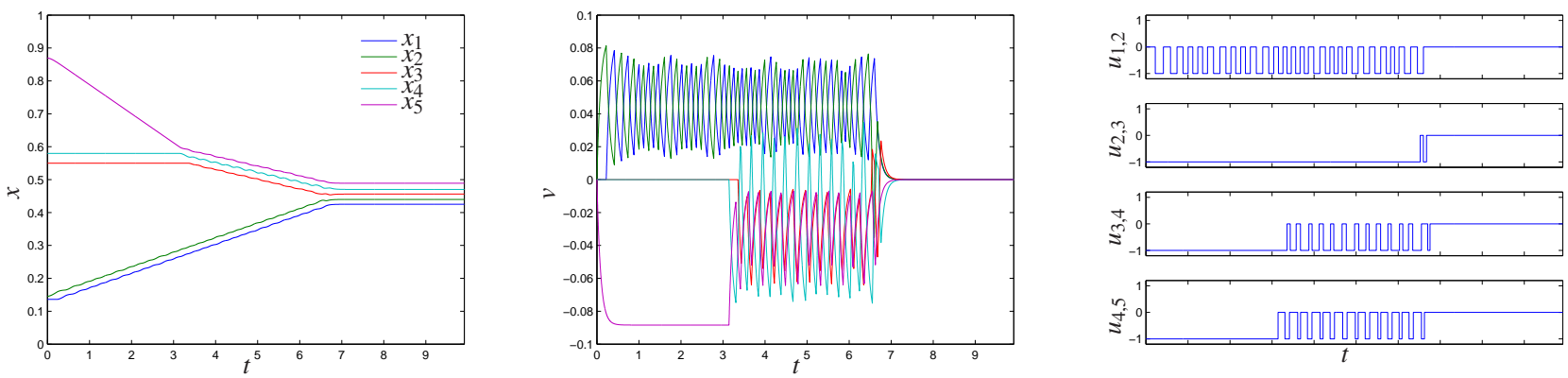

Fig. 2. Simulation of system (5), (6) on a line graph with $n=5$ nodes, $\varepsilon=0.02$, and $g=5.65$. Condition on $g$ in Theorem 1 is not satisfied. Initial positions in $[0,1]$, null initial velocities.
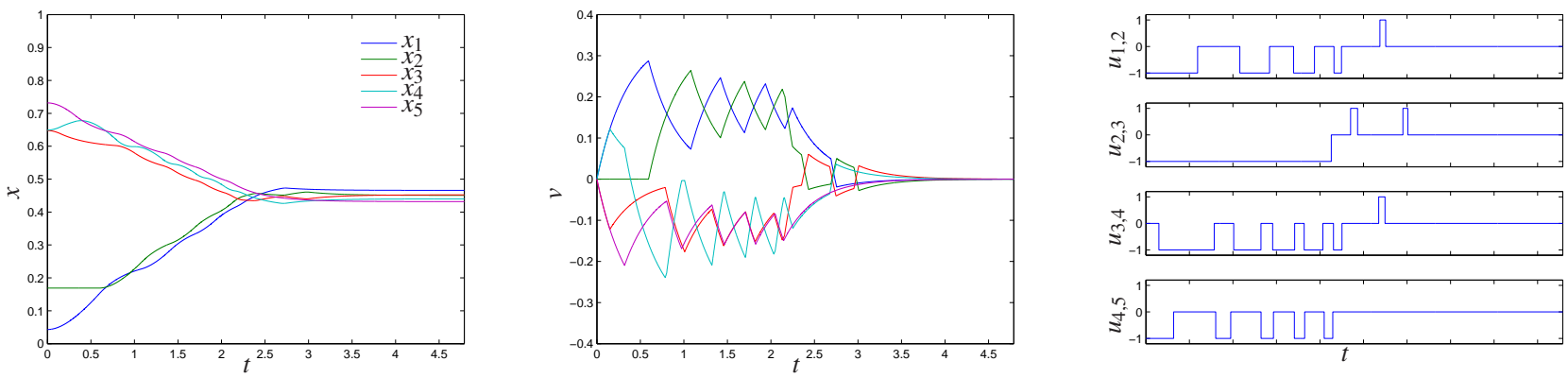

Fig. 3. Simulation of system (5), (6) on a line graph with $n=5$ nodes, $\varepsilon=0.02$, and $g=1.41$. Condition on $g$ in Theorem 1 is not satisfied. Initial positions in $[0,1]$, null initial velocities.

[11] L. Moreau." Stability of multiagent systems with time-dependent communication links". IEEE Transactions on Automatic Control, vol. 50, no. 2, pp. 169-182, 2005.

[12] C. Nowzari and J. Cortés, "Self-triggered coordination of robotic networks for optimal deployment," Automatica, vol. 48, no. 6, pp. 1077-1087, 2012.

[13] R. Postoyan, A. Anta, D. Nešić, P. Tabuada. "A unifying Lyapunovbased framework for the event-triggered control of nonlinear systems". In Proceedings of the 2011 50th IEEE Conference on Decision and Control and European Control Conference (CDC-ECC), Orlando, FL, USA, December 12-15, 2011, pp. 2559-2564.

[14] J. Qin, H. Gao, W.X. Zheng. "Second-order consensus for multi-agent systems with switching topology and communication delay". Systems $\mathcal{E}$ Control Letters, vol. 60, no. 6, pp. 390-397, 2011.

[15] R. Olfati-Saber, J.A. Fax, and R.M. Murray. "Consensus and cooperation in networked multi-agent systems". Proceedings of the IEEE, vol. 95 , no. 1 , pp. $215-233,2007$.
[16] W. Ren, R. Beard. Distributed Consensus in Multi-vehicle Cooperative Control: Theory and Applications. Communications and Control Engineering. Springer, 2007.

[17] W. Ren, R.W. Beard, and E.M. Atkins. "Information consensus in multivehicle cooperative control". IEEE Control and Systems Magazine, vol. 27, no. 2, pp. 71-82, 2007.

[18] G. Seyboth, D. Dimarogonas, and K. Johansson, "Event-based broadcasting for multi-agent average consensus," Automatica, vol. 49, no. 1, pp. 245-252, 2013.

[19] H. Yu and P.J. Antsaklis, "Event-Triggered Output Feedback Control for Networked Control Systems using Passivity: Triggering Condition and Limitations. In Proceedings of the 2011 50th IEEE Conference on Decision and Control and European Control Conference (CDC-ECC), Orlando, FL, USA, December 12-15, 2011, pp. 199-204. 


\section{APPENDIX}

Proof of Lemma 1: During continuous evolution

$$
\frac{d}{d t}\left(p_{j}(t)-p_{i}(t)\right)=v_{j}(t)-v_{i}(t)
$$

and since the state $p$ does not change during discrete transitions, the above equation holds for almost all $t$ for which the solution to the hybrid algorithm exists. On the other hand,

$$
\begin{aligned}
\frac{d}{d t}\left(v_{j}(t)\right. & \left.-v_{i}(t)\right)= \\
& -2 g\left(v_{j}(t)-v_{i}(t)\right)+\sum_{\ell=1}^{n} a_{j \ell} u_{j}^{\ell}(t)-\sum_{m=1}^{n} a_{i m} u_{i}^{m}(t)
\end{aligned}
$$

By integration of (16), we obtain the convolution equation

$$
\begin{aligned}
v_{j}(t)-v_{i}(t) & =\mathrm{e}^{-2 g t}\left(v_{j}(0)-v_{i}(0)\right) \\
+ & \int_{0}^{t} \mathrm{e}^{-2 g(t-s)}\left(\sum_{\ell=1}^{n} a_{j \ell} u_{j}^{\ell}(s)-\sum_{m=1}^{n} a_{i m} u_{i}^{m}(s)\right) d s
\end{aligned}
$$

The triangle inequality yields

$$
\begin{aligned}
& \left|v_{j}(t)-v_{i}(t)\right| \leq\left|v_{j}(0)-v_{i}(0)\right| \\
& \quad+\int_{0}^{t} \mathrm{e}^{-2 g(t-s)}\left|\sum_{\ell=1}^{n} a_{j \ell} u_{j}^{\ell}(s)-\sum_{m=1}^{n} a_{i m} u_{i}^{m}(s)\right| d s \\
& \quad \leq\left|v_{j}(0)-v_{i}(0)\right|+\left(d_{j}+d_{i}\right) \int_{0}^{t} \mathrm{e}^{-2 g(t-s)} d s \\
& \quad \leq\left|v_{j}(0)-v_{i}(0)\right|+\frac{d_{j}+d_{i}}{2 g}
\end{aligned}
$$

This bound implies that for all $t$,

$$
f_{i}^{j}(p(t), v(t)) \geq \frac{\varepsilon}{2\left(\left|v_{i}(0)-v_{j}(0)\right|+\frac{d_{i}+d_{j}}{2 g}\right)},
$$

and in particular that (8) holds, which proves (i). Bearing in mind (15), we have

$$
\begin{aligned}
\left|p_{j}(t)-p_{i}(t)\right| \geq \mid & p_{j}\left(t_{m}^{i j}\right)-p_{i}\left(t_{m}^{i j}\right) \mid \\
& -\left(\left|v_{j}\left(t_{m}^{i j}\right)-v_{i}\left(t_{m}^{i j}\right)\right|+\frac{d_{i}+d_{j}}{2 g}\right)\left(t-t_{m}^{i j}\right) .
\end{aligned}
$$

If $\left.\mid p_{j}\left(t_{m}^{i j}\right)-p_{i}\left(t_{m}^{i j}\right)\right) \mid \geq \varepsilon$, then

$$
\begin{aligned}
\left|p_{j}(t)-p_{i}(t)\right| \geq & \left|p_{j}\left(t_{m}^{i j}\right)-p_{i}\left(t_{m}^{i j}\right)\right| \\
& -\left(\left|v_{j}\left(t_{m}^{i j}\right)-v_{i}\left(t_{m}^{i j}\right)\right|+\frac{d_{i}+d_{j}}{2 g}\right)\left(t-t_{m}^{i j}\right) \\
\geq & \left|p_{j}\left(t_{m}^{i j}\right)-p_{i}\left(t_{m}^{i j}\right)\right| \\
& -\left(\left|v_{j}\left(t_{m}^{i j}\right)-v_{i}\left(t_{m}^{i j}\right)\right|+\frac{d_{i}+d_{j}}{2 g}\right)\left(t_{m+1}^{i j}-t_{m}^{i j}\right) \\
\geq & \frac{\left|p_{j}\left(t_{m}^{i j}\right)-p_{i}\left(t_{m}^{i j}\right)\right|}{2} .
\end{aligned}
$$

Moreover, similar arguments show that for $t \in\left[t_{m}^{i j}, t_{m+1}^{i j}\right]$ the sign of $p_{j}(t)-p_{i}(t)$ does not change, which proves (ii).

Proof of Lemma 2: Preliminarily, we observe that the definition of $\beta_{i j}(p(t))$ is well-posed because by Lemma 1, if $\left|p_{j}\left(t_{m}^{i j}\right)-p_{i}\left(t_{m}^{i j}\right)\right| \geq \varepsilon$, then $\left|p_{j}(t)-p_{i}(t)\right| \neq 0$ for all $t \in\left[t_{m}^{i j}, t_{m+1}^{i j}\right]$.
As a consequence of $a_{i i}=0$ for all $i \in I$, we have $\beta_{i i}(p(t))=0$ for all $i \in I$.

Note that by the sign preservation property stated in Lemma 1,

$$
\begin{gathered}
\operatorname{sign}_{\varepsilon}\left(p_{j}\left(t_{m}^{i j}\right)-p_{i}\left(t_{m}^{i j}\right)\right)=\frac{\operatorname{sign}_{\varepsilon}\left(p_{j}\left(t_{m}^{i j}\right)-p_{i}\left(t_{m}^{i j}\right)\right)}{p_{j}(t)-p_{i}(t)} . \\
\cdot\left(p_{j}(t)-p_{i}(t)\right)=\frac{1}{\left|p_{j}(t)-p_{i}(t)\right|}\left(p_{j}(t)-p_{i}(t)\right) .
\end{gathered}
$$

Hence, system (10) rewrites as

$$
\begin{aligned}
& \dot{p}_{i}(t)=v_{i}(t) \\
& \dot{v}_{i}(t)=-2 g v_{i}(t)+\sum_{j=1}^{n} \beta_{i j}(p(t))\left(p_{j}(t)-p_{i}(t)\right) .
\end{aligned}
$$

Consider now the change of coordinates (11). This returns the system

$$
\begin{aligned}
& \dot{p}_{i}(t)=g\left(y_{i}(t)-p_{i}(t)\right) \\
& \dot{y}_{i}(t)=g\left(p_{i}(t)-y_{i}(t)\right)+\frac{1}{g} \sum_{j=1}^{n} \beta_{i j}(p(t))\left(p_{j}(t)-p_{i}(t)\right) .
\end{aligned}
$$

A straightforward manipulation yields

$$
\begin{aligned}
\dot{p}_{i}(t)= & g\left(y_{i}(t)-p_{i}(t)\right) \\
\dot{y}_{i}(t)= & \left(g-\frac{1}{g} \sum_{j=1}^{n} \beta_{i j}(p(t))\right)\left(p_{i}(t)-y_{i}(t)\right) \\
& +\frac{1}{g} \sum_{j=1}^{n} \beta_{i j}(p(t))\left(p_{j}(t)-y_{i}(t)\right) .
\end{aligned}
$$

If for $i \in\{1, \ldots, n\}$ we define the variables $x_{i}$ as in (12), then system (20) rewrites as system (13).

By Lemma 1, if $\left|p_{j}\left(t_{m}^{i j}\right)-p_{i}\left(t_{m}^{i j}\right)\right| \geq \varepsilon$ then $\left|p_{j}(t)-p_{i}(t)\right| \geq$ $\frac{\varepsilon}{2}$ for all $t \in\left[t_{m}^{i j}, t_{m+1}^{i j}\right]$. This implies that $\beta_{i j}(p(t)) \leq \frac{2}{\varepsilon} a_{i j}$. Hence, provided $g \geq \sqrt{\frac{2 d_{i}}{\varepsilon}}$ for each $i$, then $g$ is such that $g-\frac{1}{g} \sum_{j=1}^{n} \beta_{i j}(p(t)) \geq 0$ for all $i \in I$ and $t \geq 0$, and all the coefficients $\alpha$ in (13) are non-negative. We also notice that $a_{i j}=a_{j i}$ implies $\beta_{i j}(p(t))=\beta_{j i}(p(t))$ for all $t$. Then, as in the proof of Theorem 1 in [14], one can notice that the graph $\tilde{\mathcal{G}}$ associated with the system (13) is weight balanced. As a matter of fact, for $i \in\{1, \ldots, n\}$,

$$
\sum_{j=1}^{2 n} \alpha_{2 i-1, j}(t)=\alpha_{2 i-1,2 i}(t)=g .
$$

On the other hand,

$$
\begin{aligned}
\sum_{j=1}^{2 n} \alpha_{j, 2 i-1}(t) & =\sum_{j=1}^{n} \alpha_{2 j, 2 i-1}(t)+\sum_{j=1}^{n} \alpha_{2 j-1,2 i-1}(t) \\
& =\sum_{j=1, j \neq i}^{n} \alpha_{2 j, 2 i-1}(t)+\alpha_{2 i, 2 i-1}(t) \\
& =\sum_{j=1, j \neq i}^{n} \frac{1}{g} \beta_{i j}(x(t))+g-\frac{1}{g} \sum_{j=1, j \neq i}^{n} \beta_{i j}=g .
\end{aligned}
$$

Similarly one shows that $\sum_{j=1}^{2 n} \alpha_{2 i, j}(t)=\sum_{j=1}^{2 n} \alpha_{j, 2 i}(t)=g$ for all $i \in\{1, \ldots, n\}$. Finally, since the system is of the form (13), there holds $\left|x_{h}(t)\right| \leq \max _{k}\left|x_{k}(0)\right|$ for all $t$. In particular, all $p_{i}(t)$ remain in some compact set dependent on the initial conditions, and it follows from the definition of $\beta_{i j}$ that either $\beta_{i j}(t)=0$ or $\beta_{i j} \geq \frac{\max _{i j} a_{i j}}{2 \max _{k}\left|x_{k}(0)\right|}=: \beta_{0}$. 